Service social

\title{
Charles-Édouard Bourgeois et Gonzalve Poulin. Deux visions différentes des services à l'enfance dans le Québec des années quarante.
}

\section{Renée Joyal et Carole Chatillon}

Volume 42, numéro 3, 1993

Perspectives théoriques

URI : https://id.erudit.org/iderudit/706634ar

DOI : https://doi.org/10.7202/706634ar

Aller au sommaire du numéro

Éditeur(s)

École de service social de l'Université Laval

ISSN

1708-1734 (numérique)

Découvrir la revue

Citer cette note

Joyal, R. \& Chatillon, C. (1993). Charles-Édouard Bourgeois et Gonzalve Poulin Deux visions différentes des services à l'enfance dans le Québec des années quarante. Service social, 42(3), 137-149. https://doi.org/10.7202/706634ar
Résumé de l'article

Une grande effervescence caractérise le Québec des années quarante. La croissance et l'urbanisation massives de la population, de même que les traumatismes engendrés par la crise économique des années trente, entraînent de profonds bouleversements sociaux qui se répercutent sur la vie familiale et la situation des enfants. Dans ce contexte inédit, deux courants de pensée s'affrontent: l'un, traditionaliste, mise sur la consolidation et l'amélioration des institutions en place, très majoritairement dirigées par des religieux; l'autre, moderniste, préconise une plus grande intervention de l'État dans le domaine social et une diversification des services. Les points de vue de l'abbé Bourgeois et du père Poulin illustrent bien ces deux visions, qui trouveront notamment à s'exprimer au sujet de la Loi de la protection de l'enfance de 1944, adoptée sous le gouvernement libéral d'Adélard Godbout, mais subséquemment mise à l'écart sous le gouvernement unioniste de Maurice Duplessis. 


\title{
NOTE HISTORIQUE
}

\section{Charles-Édouard Bourgeois et Gonzalve Poulin Deux visions différentes des services à l'enfance dans le Québec des années quarante*}

Renée JOYAL

Avocate et professeure Département des sciences juridiques Université du Québec à Montréal

Carole CHATILLON

Avocate et agente de recherche Université du Québec à Montréal

\begin{abstract}
Au cours des quinze dernières années, des changements importants sont intervenus au Québec dans le domaine de la protection de l'enfance et de la jeunesse. L'adoption de la Loi sur la protection de la jeunesse' et la mise en place de l'imposant appareil administratif destiné à en assurer l'application en témoignent. Les nombreux groupes de travail, comités et commissions constitués par le gouvernement québécois pour examiner le fonctionnement de la loi et y proposer des améliorations illustrent également les efforts soutenus de l'État en la matière ${ }^{2}$. Cet enjeu social majeur reçoit donc, depuis plus $d^{\prime}$ une décennie, une attention constante de la part d'un grand nombre de personnes de formations et $d^{\prime}$ occupations diverses.
\end{abstract}

* Ce texte a été élaboré et rédigé dans le cadre d'un projet de recherche subventionné par le Conseil de recherches en sciences humaines du Canada et dirigé par Renée Joyal. Louise Chauvette, M.A. (Histoire), a largement contribué à la recherche documentaire. 
Bien que le passé récent ait été particulièrement fécond en recherches théoriques et pratiques sur la question, il est une autre période de I'histoire québécoise qui connut une effervescence remarquable à ce chapitre. Il s'agit des années trente et quarante. Deux commissions d'enquête ${ }^{3}$ se sont alors penchées sur le phénomène de l'enfance malheureuse et l'on assista, en 1944, à l'adoption d'une première loi moderne de protection de l'enfance ${ }^{4}$. Cette loi suscita toutefois une opposition très vive dans une bonne partie du monde religieux et politique; le retour au pouvoir de Maurice Duplessis, quelques mois plus tard ${ }^{5}$, assura pour un temps la prédominance des forces conservatrices et entraîna, par le fait même, la mise à l'écart de la loi.

Parmi toutes les personnes qui s'intéressaient à ce domaine à l'époque se distinguent deux religieux, l'abbé Charles-Édouard Bourgeois et le père Gonzalve Poulin. Le premier, que l'on surnomme "I'ami des enfants abandonnés", dirige à Trois-Rivières la Centrale des œuvres de bienfaisance à l'enfance ${ }^{6}$. II exerce une grande influence sur le clergé et l'épiscopat catholiques, de même qu'auprès de Maurice Duplessis ${ }^{7}$, dont il sera le conseiller principal en matière de protection de l'enfance tant durant les années d'opposition que de pouvoir de cet homme politique. Ses écrits ${ }^{8}$ révèlent une vision traditionaliste de la problématique de la protection de l'enfance. Le second, le père Gonzalve Poulin, manifeste tout au long de sa carrière un intérêt marqué pour la famille ${ }^{9}$. Ses propos abondants sur ce thème jettent un éclairage non équivoque sur sa perception du problème de l'enfance malheureuse. Le point de vue qu'il adopte est nettement plus moderniste que celui de l'abbé Bourgeois. Ses études, son enseignement et son apostolat social témoignent $d^{\prime}$ 'une volonté marquée de sortir des sentiers battus pour répondre efficacement aux problèmes de I'heure.

La richesse de l'œuvre de ces deux religieux et leur vision respective de la protection de $\mathrm{l}^{\prime}$ enfance ${ }^{10}$ méritent une attention particulière. Le rappel du parcours biographique de chacun d'eux et de leurs opinions souvent divergentes sur les questions familiales et sociales permet de mieux saisir les enjeux de la protection de l'enfance dans le Québec français d'alors.

\section{DES VIES CONSACRÉES AUX PROBLÈMES DE L'ENFANCE ET DE LA FAMILLE}

Les deux pionniers que furent l'abbé Bourgeois et le père Poulin sont probablement parmi les premières personnes au Québec à avoir reçu une formation universitaire poussée en relation avec leur travail dans le domaine de l'enfance et de la famille. 


\section{La poursuite d'études spécialisées}

En 1931, au moment où il est nommé aumônier de l'Orphelinat SaintDominique $^{11}$, l'abbé Bourgeois a trente-trois ans. C'est le début de sa carrière auprès des enfants abandonnés ${ }^{12}$. Le père Poulin, lui, a vingt-cinq ans. Il est ordonné prêtre l'année suivante, en 1932, après des études philosophiques et théologiques chez les Franciscains ${ }^{13}$.

Le cheminement universitaire des deux hommes diffère sensiblement. Homme d'action beaucoup plus que théoricien, c'est seulement à l'âge de quarante-six ans que Charles-Édouard Bourgeois termine ses études de doctorat; il soutient alors une thèse intitulée La protection de I'enfance dans la province de Québec ${ }^{14}$. Les études de Gonzalve Poulin suivent un cours plus régulier; il s'y consacre sans répit. Après des études en journalisme et en sciences sociales au Québec, il se rend en France afin de parfaire sa formation. Il obtient son doctorat en 1938, à l'âge de trente-deux ans, après avoir soutenu une thèse portant sur l'éducation des adultes en Angleterre ${ }^{15}$.

II n'existe alors aucun programme universitaire de service social en milieu canadien-français ${ }^{16}$. Pourtant, la société québécoise fait face à des problèmes inédits qui appellent des modes d'intervention différents de ceux qui sont alors pratiqués. Jusque-là, I'entraide familiale et paroissiale a suffi à soulager tant bien que mal les misères humaines. Mais le Québec des années trente est en pleine crise économique. Chômage et pauvreté frappent de plein fouet les familles québécoises et entraînent de graves problèmes de désorganisation familiale et sociale. Mis à part les services fournis par les Sociétés de Saint-Vincent-de-Paul, il n'existe pratiquement aucune forme d'aide à domicile en milieu canadien-français ${ }^{17}$. Les prestations sociales sont inexistantes ${ }^{18}$. Les enfants dont les besoins primaires ne peuvent plus être satisfaits dans leurs familles sont placés en institution, une solution couramment utilisée pour compenser le manque à gagner des parents ${ }^{19}$.

Les deux religieux s'attaqueront, chacun à leur manière, aux problèmes du moment.

\section{Un engagement apostolique exemplaire}

Il convient de parler d'engagement " apostolique » plutôt que d'engagement «professionnel» pour qualifier les œuvres de l'abbé Bourgeois et du père Poulin. Il est clair que leur action dans le domaine de l'enfance et de la famille est indissociable de leur vocation sacerdotale, qu'elle en est même le mode d'accomplissement privilégié. Dans le cas de l'abbé Bourgeois d'ailleurs, nous aurons l'occasion de relater plus loin ses 
réticences à la « laïcisation » des services à l'enfance et à la famille, réticences que ne semble pas partager le père Poulin.

Si les études avancées de celui-ci le préparent bien à l'enseignement et à l'écriture, il est une autre activité à laquelle il consacre une bonne part de son temps: la fondation d'organismes sociaux. Le père Poulin mènera de front les trois volets de sa carrière. Ainsi, dès son retour de France où il vient d'obtenir son doctorat, il s'installe à Montréal et collabore activement aux revues Culture ${ }^{20}$ et La Famille L $^{21}$ Il donne des cours à l'École supérieure de pédagogie familiale et à I'Institut Pie IX ${ }^{22}$; en 1939, il fonde I'association "Les Amis de la famille», dont le but est d'organiser des conférences et des journées familiales; puis, en 1942, il met sur pied I'Institut familial, centre d'études, d'enquête et d'apostolat où sont donnés, entre autres, des cours et des conférences sur la famille ${ }^{23}$.

En 1943, le père Georges-Henri Lévesque, fondateur de l'École, puis de la Faculté des sciences sociales de I'Université Laval, I'invite à faire partie de son équipe. II devient directeur-fondateur de la nouvelle École de service social reliée à cette faculté, fonction qu'il occupera jusqu'en $1956^{24}$. Il y donne plusieurs cours, notamment de sociologie et de morale familiales, de philosophie et $d^{\prime}$ 'histoire du service social ${ }^{25}$.

II participe à la fondation de plusieurs organismes de soutien à la famille, dont le Service familial de Québec et le Conseil central des œuvres, devenu maintenant Centraide. En 1951, il met sur pied la revue Service social ${ }^{26}$. Dans le cadre des travaux de la Commission royale d'enquête sur les problèmes constitutionnels mise sur pied dans les années cinquante, il rédige une étude importante qui retrace l'évolution de I'assistance sociale dans la province de Québec de 1608 à $1951^{27}$.

Charles-Édouard Bourgeois, comme on l'a vu plus haut, est surtout un homme $d^{\prime}$ action. Ses énergies sont consacrées principalement au développement d'œuvres de bienfaisance dans la région de Trois-Rivières. Ainsi, dès après sa nomination, en 1931, au poste d'aumônier de l'Orphelinat Saint-Dominique, il s'emploie activement à mettre sur pied des services variés pour répondre aux besoins des enfants orphelins ou sans soutien de cette région. Crèches, orphelinats, écoles maternelles, centres $d^{\prime}$ apprentissage agricole et industriel, écoles ménagères, autant d'institutions qu'il crée si elles n'existent pas, ou qu'il restructure si elles sont déjà en place. Des colonies de vacances, un service d'adoption et de placement des orphelins ayant reçu une formation technique complètent le tout. Pour intégrer ces divers éléments et leur donner une plus grande efficacité, il met en place une Centrale qui dispose notamment d'un service d'enquête et de classification. En 1939, I'organisme prendra le nom de "L'assistance à l'enfant sans soutien ${ }^{28}$ ".

En 1934, l'abbé Bourgeois fonde le Centre de service social du diocèse des Trois-Rivières. En 1948, il mettra sur pied I'Institut psycho-social 
de la même ville ${ }^{29}$. Il ne se contente pas de créer et de diriger des œuvres de bienfaisance. II participe également à des rencontres internationales sur le thème de la protection de l'enfance et de la jeunesse. C'est ainsi qu'il effectue plusieurs voyages en Europe à titre de délégué officiel de la Province de Québec et de la Conférence catholique canadienne ${ }^{30}$.

II n'hésite pas, par ailleurs, à prendre position, au Québec même, sur la question controversée de la protection de I'enfance. À l'occasion, notamment, des audiences publiques tenues en 1944 par la Commission d'assurance-maladie de Québec, il fait connaître son point de vue sur plusieurs aspects du problème, dont l'adoption, le placement en foyer nourricier et la professionnalisation des services d'aide à l'enfance et à la famille. Son point de vue diverge sensiblement de celui exprimé par le père Poulin dans ses nombreux écrits.

\section{DES OPINIONS DIVERGENTES SUR LES QUESTIONS FAMILIALES ET SOCIALES}

Les points de vue divergents de l'abbé Bourgeois et du père Poulin sur de nombreuses facettes de l'épineuse question de la protection de l'enfance sont tributaires de visions diamétralement opposées de la place respective de l'Église et de l'État dans le champ du bien-être social.

\section{Le bien-être social, I'Église et l'État}

$\mathrm{Si}$, pour Charles-Édouard Bourgeois, I'assistance sociale doit demeurer avant tout une affaire de charité chrétienne où le bien-être spirituel de I'homme occupe la première place ${ }^{31}$, Gonzalve Poulin, lui, croit que l'Église doit limiter "son action charitable à un rôle supplétif dans tous les grands services d'assistance à domicile et d'hospitalisation ", que sans renoncer à sa mission de charité, elle doit collaborer avec l'État à travers "des formes d'action charitable plus conformes à son esprit ${ }^{32}$ ».

Un désengagement relatif de l'Église du champ du bien-être social ne semble donc inspirer aucune crainte au père Poulin; I'abbé Bourgeois, pour sa part, y voit de graves dangers. Ainsi, bien qu'il déplore le manque de fonds alloués à la formation du personnel en ce domaine, CharlesÉdouard Bourgeois craint la spécialisation des services à l'enfance, en raison de la laïcisation probable de ceux-ci par la suite et du glissement de «nos sociétés charitables vers les gouffres du matérialisme ${ }^{33}$ ». De son côté, Gonzalve Poulin exhorte les laïcs à s'impliquer activement dans le gouvernement de l'Église pour éviter que ne persiste l'anticléricalisme, " cette opposition sournoise et parfois ouverte contre les influences indues du clergé et ses ambitions jugées trop terrestres ou démesurées ${ }^{34}$ ». 
Mais il n'y pas que la primauté de la vie spirituelle qui risque d'être affectée par la laïcisation des structures de bien-être social, pense l'abbé Bourgeois. Une présence accrue des pouvoirs publics constitue également une menace à l'autonomie des œuvres d'assistance à l'enfance, dont les responsables, selon lui, doivent jouir d'une complète liberté d'action ${ }^{35}$. S'il craint « la mainmise de l'État sur les œuvres de charité», il réclame par ailleurs que celui-ci prenne à sa charge tous les frais d'assistance publique et finance l'agrandissement des bâtiments existants, sans que cette aide financière affecte, par ailleurs, les prérogatives de l'Église dans ce domaine ${ }^{36}$.

Voilà donc exposées deux visions différentes du rôle respectif de l'Église et de l'État dans le champ du bien-être social. D'une part, une reconnaissance de la légitimité pour l'État de prendre charge de ce secteur même si cela " exigera peut-être le retrait progressif de l'Église de la direction des grandes institutions $d^{\prime}$ assistance ${ }^{37}$ » et, $d^{\prime}$ autre part, le maintien de la position dominante de l'Église dans ce domaine pour sauvegarder la primauté de la vie spirituelle et assurer l'autonomie des institutions en place.

Ces options idéologiques fondamentales de l'abbé Bourgeois et du père Poulin colorent, évidemment, les opinions qu'ils expriment sur divers aspects de la Loi de la protection de l'enfance de 1944.

\section{La protection de l'enfance}

$C^{\prime}$ est à la suite du rapport de la Commission d'assurance-maladie de Québec $^{38}$, connu sous le nom de Rapport Garneau, que l'Assemblée législative est appelée à se pencher sur un nouveau texte de loi en matière de protection de l'enfance. On se rappelle que la Commission Garneau, mise sur pied en 1943 pour étudier l'établissement éventuel d'un régime d'assurance-maladie pour le Québec, se voit aussi confier par la suite le mandat de faire enquête sur le problème des garderies et de la protection de l'enfance ${ }^{39}$. La troisième partie du Rapport Garneau énonce un avantprojet de loi en la matière. La Loi concernant les écoles d'industrie, en vigueur à l'époque, ne prévoyait que le placement institutionnel comme remède aux problèmes de l'enfance malheureuse; par ailleurs, le recours à l'appareil judiciaire, seul mécanisme d'application de la loi, s'exerçait selon une procédure très sommaire ${ }^{40}$.

Le nouveau texte, présenté le 10 mai 1944 à l'Assemblée législative et intitulé Loi concernant la protection de l'enfance ${ }^{41}$, rompt avec ce passé ; il considère la question de façon globale et $n^{\prime}$ est plus centré sur le placement des enfants concernés dans des institutions d'assistance.

On y retrouve donc des changements radicaux par rapport à la législation antérieure, notamment sur le plan des nouvelles responsabilités que 
se reconnaît l'État en matière de protection de l'enfance. Celles-ci trouvent à s'exercer à travers divers mécanismes étroitement reliés. Les fonctions de réception, d'orientation et de prise en charge des situations de négligence sont confiées à des sociétés de protection de l'enfance régionales, alors que le pouvoir de trancher les litiges est réservé à un tribunal spécialisé. La coordination et la supervision des sociétés et des institutions relèvent du Conseil supérieur et du directeur de la protection de l'enfance ${ }^{42}$.

Plusieurs dispositions de ce texte législatif suscitent chez CharlesÉdouard Bourgeois une très vive opposition. II s'en prend d'abord à la composition du Conseil supérieur de la protection de l'enfance, organisme réunissant douze membres, dont dix de religion catholique et deux de religion protestante. Selon lui, le fait de faire siéger catholiques et protestants au sein d'une même instance remet en question le principe du caractère confessionnel de nos institutions. II propose qu'on s'inspire à cet égard de la formule qui prévaut dans le domaine de l'instruction publique où existent un comité catholique et un comité protestant ${ }^{43}$.

La proposition législative prévoit également que les enfants concernés puissent demeurer dans leur famille sous la supervision d'une société ou être placés en institution, en foyer nourricier, en apprentissage ou en service domestique par les soins d'une telle société. Cet élargissement des mesures susceptibles d'être ordonnées par le tribunal et leur suivi par des sociétés dont la constitution relève de l'autorité civile et non de l'autorité religieuse préoccupent grandement I'abbé Bourgeois. II aurait préféré "que nos évêques déterminent eux-mêmes la part délicate que devra (sic) ou pourra (sic) jouer notre clergé et nos laïques dans ces nouvelles sociétés... ou encore quels pouvoirs pourront exercer ces mêmes organismes ». Charles-Édouard Bourgeois ne cache pas sa préférence pour le placement institutionnel des enfants malheureux. II croit cette formule garante des valeurs chrétiennes, à la fois parce qu'elle assure la formation morale et religieuse de l'enfance et qu'elle ne porte pas atteinte à l'influence des autorités religieuses qui dirigent ces institutions ${ }^{44}$.

Appelé à présenter son point de vue aux auditions de la Commission Garneau, Charles-Édouard Bourgeois avait d'ailleurs affirmé qu'il ne voyait pas la nécessité d'une nouvelle loi, mais que, si une telle loi était adoptée, elle devrait demeurer "sous l'égide tutélaire de l'Église et le contrôle bienveillant de l'État ${ }^{45}$ ». Dans un article publié subséquemment, il qualifie le texte législatif de loi «malheureuse » et "dangereuse » et considère que c'est "pour calmer les nouveaux sociologues de l'enfance »

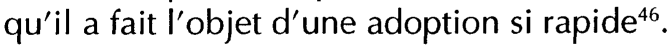

Aucun document n'a pu être retracé faisant état de commentaires explicites de la part de Gonzalve Poulin sur la proposition législative. Celui-ci reconnaît cependant l'insuffisance des lois de protection de 
I'enfance à cette époque et se prononce clairement en faveur de l'instruction publique obligatoire jusqu'à seize ou dix-huit ans ${ }^{47}$.

Le Service familial de Québec, dont il est membre-fondateur en 1943, présente pour sa part un mémoire devant la Commission Garneau ${ }^{48}$. On peut supposer que le point de vue de cet organisme rejoint sensiblement celui de Gonzalve Poulin, d'autant plus que le personnel qui y travaille est en grande partie diplômé de l'École de service social de I'Université Laval, qu'il dirige.

D'entrée de jeu, les porte-parole de l'organisme réclament une législation de protection efficace pour "l'enfant abandonné, en détresse, en danger moral ou physique ». Ils tiennent à ce que l'État prenne ses responsabilités dans ce domaine et mette en place des services appropriés aux besoins des enfants, soit en offrant lui-même ces services ou en octroyant des subventions à des organismes chargés de les offrir ; I'essentiel étant, selon le Service familial de Québec, que le tout soit fait selon " les standards reconnus » et par le truchement d'un personnel spécialisé, formé avec l'aide des pouvoirs publics.

L'organisme privilégie le placement familial des enfants en difficulté, estimant que la famille restera toujours «le milieu idéal pour l'épanouissement de l'enfant ». À cette fin, le Service familial de Québec veut faire reconnaître à l'enfant le "droit à une atmosphère familiale saine, droit à I'affection qui lui donne la sécurité indispensable à l'épanouissement harmonieux de toute sa personne... ${ }^{49} »$.

Ce discours se rapproche du point de vue du père Poulin, selon qui le système institutionnel a un urgent besoin des compléments du placement familial et des techniques du service social ${ }^{50}$. Souhaitant qu'on cesse d'opposer les deux formes de placement, il estime que seul le bien intégral de l'enfant doit régir toute décision en la matière. Il considère que «le placement d'un enfant hors de son foyer naturel signifie du point de vue de son équilibre émotif et de sa stabilité sociale une catastrophe que rien ne pourra jamais complètement réparer ${ }^{51}$ »; il soutient, par ailleurs, que le placement en foyer nourricier ne présente pas de garanties légales suffisantes en l'absence d'une véritable législation sur la protection de l'enfance ${ }^{52}$.

Rappelons également qu'en 1942, à la réunion annuelle de l'Institut familial, qu'il dirige, s'élabore une Charte des droits de l'enfant, qui fait figure de programme de société pour assurer le développement intégral et harmonieux de l'enfant ${ }^{53}$.

\section{CONCLUSION}

Ce bref rappel de l'œuvre et de la pensée de l'abbé Charles-Édouard Bourgeois et du père Gonzalve Poulin révèle deux styles, deux approches. 
Le premier se fait le champion de la consolidation et de l'amélioration des services offerts aux enfants sans soutien dans le cadre de la tradition; le second croit que l'aide aux enfants malheureux passe désormais par une remise en cause du cadre traditionnel et une présence accrue de l'État ${ }^{54}$.

Cette divergence de vues peut certes s'expliquer en partie par des différences de personnalité et de cheminement. On sait que, chez l'abbé Bourgeois, action et réflexion sont intimement liées; chez le père Poulin, $d^{\prime}$ assez longues études, effectuées en partie en Europe, précèdent l'engagement social. Les milieux très différents dans lesquels furent appelés à intervenir les deux hommes ont également pu contribuer à l'élaboration de leurs points de vue respectifs. Charles-Édouard Bourgeois, installé à Trois-Rivières, connaît bien les problèmes d'une petite ville et de son environnement rural et semi-rural. Gonzalve Poulin, qui s'établit à Québec à partir de 1943, demeure toujours très actif dans les cercles montréalais préoccupés de l'aide aux enfants et aux familles ${ }^{55}$. II connaît donc les problèmes de la grande ville et $d^{\prime}$ une ville de dimension moyenne comme Québec.

Il va de soi que les questions sociales ne se présentent pas de la même façon dans ces deux milieux. D'ailleurs, on se rappelle à ce sujet que Mgr Charbonneau, archevêque de Montréal, est souvent entré en conflit avec ses collègues de l'épiscopat sur la façon de remédier aux problèmes sociaux, et notamment sur la place de l'État dans les services à l'enfance et à la famille ${ }^{56}$.

Chômage, maladie, problèmes de logement se manifestent avec plus $d^{\prime}$ acuité dans une agglomération comme Montréal, où, par ailleurs, le déracinement et le cosmopolitisme caractéristiques de la grande ville ne favorisent pas la cohésion sociale si utile en période difficile. En milieu rural, l'entraide et la solidarité peuvent plus facilement venir combler les défaillances du système économique. Des besoins autres et une structure sociale différente appellent en milieu urbain et industrialisé des solutions inédites, adaptées à ce contexte. Le père Poulin en est convaincu.

L'abbé Bourgeois, lui, y est forcément moins sensible. II faut reconnaître aussi que la Centrale trifluvienne qu'il a mise sur pied et qu'il dirige est un modèle en son genre pour l'époque ${ }^{57}$. Fort d'appuis solides dans le monde religieux et politique, Charles-Édouard Bourgeois a réussi à organiser un réseau de services variés pour recueillir et éduquer les enfants sans soutien de Trois-Rivières et de ses environs.

Chacun à leur manière, les deux hommes ont largement contribué à faire connaître la situation des enfants malheureux et à poser les jalons d'une réflexion en profondeur sur la philosophie et les modalités de leur prise en charge; ils n'ont pas non plus ménagé leur peine pour créer de multiples organismes d'aide et de soutien. 
Bien que la Loi de la protection de l'enfance de 1944 n'ait jamais été mise en application - en raison du retour au pouvoir de l'Union nationale de Maurice Duplessis quelques mois après I'adoption de celle-ci par le gouvernement libéral d'Adélard Godbout -, l'Assemblée législative adopta, en 1950, 1951 et 1959 de nouveaux textes législatifs qui accrurent l'éventail des mesures susceptibles d'être ordonnées à l'égard des enfants en besoin de protection et firent une place officielle aux agences de service social qui avaient été mises sur pied au fil des ans ${ }^{58}$. Tous ces changements, de même que la réorganisation des services effectuée dans les années soixante et soixante-dix, pavèrent la voie à l'adoption de la Loi sur la protection de la jeunesse de $1977^{59}$.

\section{Notes}

1. L.Q. 1977 , c. 20.

2. Ainsi, les années 1991 et 1992 voient la publication de trois rapports émanant d'autant de groupes d'études chargés d'examiner divers aspects de la question : ministère de la Santé et des Services sociaux, La protection sur mesure, un projet collectif, Québec, 1991 (Rapport Harvey II) ; ministère de la Santé et des Services sociaux, Un Québec fou de ses enfants, Québec, 1991 (Rapport Bouchard) ; ministère de la Santé et des Services sociaux et ministère de la Justice, La protection de la jeunesse... Plus qu'une loi, Québec, 1992 (Rapport Jasmin).

3. D'abord, la Commission des Assurances sociales de Québec, instituée en 1930 afin d'étudier l'établissement éventuel d'un système d'assurance sociale et de placement familial dans la province et le mode de législation susceptible d'être adopté à cet égard; ensuite, la Commission d'assurance-maladie de Québec, instituée en 1943 et chargée, notamment, de faire enquête sur le problème des garderies et de la protection de l'enfance. Voir à ce sujet : Renée Joyal et Carole Chatillon, "La Loi québécoise de protection de l'enfance de 1944, genèse et avortement d'une réforme... ", à paraître à la revue Histoire sociale (1994).

4. Loi concernant la protection de l'enfance, S.Q. 1944, c. 33.

5. Joyal et Chatillon, "La Loi québécoise de protection... "

6. La Centrale est un organisme qui régit les œuvres d'assistance à l'enfance dans le diocèse des Trois-Rivières. Celles-ci comprennent une crèche, trois orphelinats, un patronage et trois colonies de vacances. La Centrale supervise l'admission des enfants dans l'une de ces institutions, leur retour au sein de leur famille ou leur adoption, légale ou de fait. Voir à ce sujet : Charles-Édouard Bourgeois, Une richesse à sauver, l'enfant sans soutien, Trois-Rivières, Éditions du bien public, 1947, p. 208.

7. L'influence qu'exerce l'abbé Bourgeois sur Maurice Duplessis s'explique en partie par les liens privilégiés qui unissent les deux hommes. Ceux-ci se connaissent de longue date, puisque le père de Charles-Édouard Bourgeois, John Bourgeois, ingénieur et arpenteur-géomètre, agit à titre d'expert pour Maurice Duplessis, alors que celui-ci exerce le droit aux Trois-Rivières. Fort de cette amitié, de même que de ses connaissances et de ses réalisations dans le domaine, l'abbé Bourgeois devient rapidement "I'homme de confiance de l'épiscopat en matière de bien-être social " (Robert Rumilly, Maurice Duplessis et son temps, t. 1, Montréal, Fides, 1973, p. 172 et 668). 
8. Parmi ceux-ci, il convient d'abord de mentionner la thèse qu'il soutient pour obtenir son grade de docteur en philosophie. Elle est publiée en 1947 sous le titre Une richesse à sauver : l'enfant sans soutien. L'ouvrage connaît deux éditions et une traduction anglaise (Biographies canadiennes-françaises, $18^{\mathrm{e}}$ édition, Montréal, J.-A. Fortin, 1960, p. 23).

9. " ...l'éternel problème qui lui tenait à cœur... Pour lui, la famille... c'était vraiment la cellule sociale. Mais une vraie cellule, c'est-à-dire une chose vivante et dynamique et non quelque chose d'inerte et de figé. Non pas une institution refermée sur elle-même, mais sans cesse ouverte... aux mutations qu'imposent de nouvelles conditions économiques, sociales et culturelles. "Extrait de l'allocution d'ouverture du père Georges-Henri Lévesque à un colloque sur la famille tenu les 11 et 12 juin 1976 à I'Université Laval, en hommage à Gonzalve Poulin. Propos rapportés dans Chroniques et documents (publication des Pères franciscains), vol. 29, n² 2, décembre 1976, p. 78.

10. Gonzalve Poulin en fait d'ailleurs état de manière explicite dans un article où il commente le livre de Charles-Édouard Bourgeois, précédemment cité. Traitant des problèmes de l'enfance, il écrit : "Les traditionalistes ne voient que l'institution comme solution fondamentale à ces problèmes ; les progressistes voudraient mitiger le système institutionnel d'éléments familiaux et de techniques de service social. C'est parce qu'il appartient au premier groupe que l'abbé Bourgeois s'oppose au placement familial "... "Le livre de Bourgeois, par les bonnes idées qu'il aura semées, ne peut que promouvoir ce progrès légal de la protection de l'enfance en dépit (...) de ses conceptions trop liées au droit existant... ": Gonzalve Poulin, "Bourgeois, Abbé Charles-Édouard, Une richesse à sauver : I'enfant sans soutien ", Culture, 1947, vol. VIII, p. 216.

11. Cet orphelinat est mis sur pied en 1910 par les Sœurs dominicaines et est situé tout près de la maison des parents de Charles-Édouard Bourgeois. Ceux-ci décident d'en faire leur œuvre, y consacrant temps et argent. Le jeune Bourgeois, issu d'un milieu aisé, découvre alors un monde pour lui inconnu, celui des enfants sans famille. Voir à ce sujet : Albert Plante, "Une réussite aux Trois-Rivières ", Relations, 1944, n 48, p. 314.

12. Il avait auparavant occupé le poste de vicaire de la paroisse de Saint-Philippe des TroisRivières (Biographies canadiennes-françaises..., p. 23).

13. Simone Paré, "De Gonzalve Poulin à l'École de service social ", Chroniques et documents (publication des Pères franciscains), vol. 29, n 2, décembre 1976, p. 70.

14. Il obtient alors le grade de docteur en Philosophie, section des sciences sociales, économiques et politiques, à l'Université d'Ottawa (Biographies canadiennesfrançaises..., p. 23).

15. En 1934-1935, il poursuit des études supérieures à la Faculté des sciences sociales de I'Université de Montréal. Il part ensuite pour Lille, où il obtient, en 1937, un Diplôme supérieur en sciences sociales. On le retrouve finalement à Paris où, après des études à I'Institut catholique et à l'École libre des Hautes Études de la Sorbonne, il est reçu docteur en sciences sociales : Paré, "De Gonzalve Poulin... ", p. 70.

16. Alors que I'Université McGill a déjà son École de service social depuis 1918, la première école catholique de service social au Canada voit le jour en 1940 ; elle est annexée à l'Université de Montréal en 1942. C'est en 1943 que la Faculté des sciences sociales de l'Université Laval donne le jour à une École de service social. Voir à ce sujet: Nicole Vanier, "Aperçu historique de l'École de service social de l'Université de Montréal ", Service social, octobre 1961 et avril 1962, vol. 10, nº 3, vol. 11, n 1 , p. 95-101 et Hayda Denault, "L'insertion du service social dans le milieu canadienfrançais ", Service social, 1961 et 1962, vol. 10, no 3, vol. 11, nº 2, p. 13.

17. Gonzalve Poulin, "La socialisation des structures de bien-être, phénomène historique ", Culture, 1964, vol. XXV, p. 337.

18. Les premières interventions de l'État dans ce domaine prennent place à la fin des années trente. C'est en 1937 que le gouvernement du Québec met en place 
un programme $d^{\prime}$ assistance aux mères nécessiteuses. Les premières prestations d'assurance-chômage seront versées par le gouvernement fédéral en 1940.

19. En 1943, dans le diocèse des Trois-Rivières, plus de mille enfants font l'objet d'un tel placement; de ce nombre, 411 sont placés en raison des difficultés économiques de leurs parents (Bourgeois, Une richesse à sauver..., p. 113).

20. Revue trimestrielle publiée par les Franciscains, Culture traite de sciences religieuses et de sciences profanes. Cette revue paraît de 1940 à 1970.

21. Il s'agit d'une revue mensuelle d'action catholique publiée par les Franciscains de 1937 à 1954 et destinée à l'étude des questions familiales.

22. Paré, "De Gonzalve Poulin... ", p. 70 et 71.

23. L'association "Les amis de la famille " s'affilie alors à l'Institut familial.

24. Le père Georges-Henri Lévesque, dominicain, a fondé en 1939 l'École des sciences sociales de l'Université Laval, laquelle se transforme en Faculté des sciences sociales en 1943. Il est également l'instigateur du projet d'École de service social de cette université.

25. Paré, "De Gonzalve Poulin... ", p. 71.

26. Id.

27. L'assistance sociale dans la Province de Québec 1608-1951, Commission royale d'enquête sur les problèmes constitutionnels, annexe 2, Québec, 1955.

28. Bourgeois, Une richesse à sauver..., p. 194-211.

29. Biographies canadiennes-françaises..., p. 23.

30. En 1937, l'abbé Bourgeois se rend en France, en Belgique et en Italie pour y étudier la situation des œuvres de protection de l'enfance. De là lui vient l'idée de fonder le patronage Saint-Charles, un centre de formation qui permet aux jeunes de terminer leurs études primaires tout en apprenant certains métiers (Plante, "Une réussite... ", p. 316). En 1947, il représente le Québec au Congrès de I'Union catholique internationale de service social, en Suisse. De 1950 à 1957, il séjourne encore plusieurs fois en Europe à l'occasion de divers congrès internationaux (Biographies canadiennesfrançaises..., p. 23). En 1952, il est nommé prélat domestique et est désigné par la suite sous le titre de monseigneur Bourgeois.

31. Ce qui est primordial, écrit-il, c'est "... que l'homme vient de Dieu et qu'il doit retourner à Dieu » : Charles-Édouard Bourgeois, "La charité et les misères humaines ", L'œuvre des tracts (brochure mensuelle publiée par I'Institut social populaire), 1951, $n^{\circ} 384$, p. 12.

32. Poulin, "La socialisation... ", p. 343 et 344.

33. Bourgeois, "La charité... », p. 1.

34. Gonzalve Poulin, "Le laïcat et I'action catholique », La Famille, 1939, t. II, n 10, p. 214. De toute évidence, les deux hommes ne voient pas " les choses terrestres " sous le même angle.

35. Il convient de rappeler qu'à cette époque 92 des 101 institutions recevant des enfants sont dirigées par des religieux ou des clercs : Bourgeois, Une richesse à sauver..., p. 240.

36. Bourgeois, "La charité... ", p. 13.

37. Poulin, "La socialisation... », p. 343.

38. Commission d'assurance-maladie de Québec, premier rapport ; la Commission, composée de MM. Antonio Garneau, avocat, Roméo Blanchet, médecin, et P.E. Durnford, homme d'affaires, avait été créée en vertu de la Loi instituant une Commission d'assurance-maladie, S.Q. 1943, c. 32. 
39. Voir à ce sujet : Joyal et Chatillon, "La Loi québécoise de protection... ".

40. Loi concernant les écoles d'industrie, S.R.Q. 1942, c. 39.

41. Loi concernant la protection de l'enfance, S.Q. 1944, c. 33.

42. Voir à ce sujet : Joyal et Chatillon, "La Loi québécoise de protection... "

43. Son témoignage a fait l'objet d'un compte rendu dans Le Devoir, 8 mars 1944, p. 3.

44. La Semaine religieuse de Québec, 58e année, n 19, 10 janvier 1946, p. 294.

45. Le Devoir, 8 mars 1944, p. 3.

46. La Semaine religieuse de Québec, 58 année, $n^{\circ} 19,10$ janvier 1946, p. 293 et 294.

47. Gonzalve Poulin, "L'enfant protégé et non exploité ", L'Éducateur, janvier 1943, p. 28 ; ce n'est que quelques mois plus tard que l'Assemblée législative adopte une loi rendant l'instruction publique obligatoire; la limite d'âge y est cependant fixée à quatorze ans.

48. Ce mémoire n'a pu être retracé. Son contenu a fait l'objet d'un compte rendu dans L'Éducateur, 1944, t. VII, p. 436.

49. Id.

50. Poulin, "Bourgeois, Abbé Charles-Édouard... », p. 216.

51. Gonzalve Poulin, "Saint-Pierre, Arthur, Témoignages sur nos orphelinats », Culture, 1946, vol. VII, p. 375.

52. Poulin, "Bourgeois, Abbé Charles-Édouard... », p. 216.

53. Cette charte est publiée dans La Famille, 1942, t. VI, $\mathrm{n}^{\circ} 1$, en troisième de couverture.

54. Les idées nouvelles que préconisait le père Poulin étaient certes difficiles à promouvoir à l'époque : "... il faut se rappeler combien était fermé le milieu dans lequel vivait la société des années trente. Tout ce qui était nouveau devenait suspect s'il n'était proposé par la hiérarchie. Tout se réglait d'autorité et les audacieux qui prenaient des initiatives personnelles, même les plus justifiées, le faisaient à leurs risques ": Richard Thivierge, "Poulin, père Gonzalve », C'était mon frère (publication des Pères franciscains), supplément $\mathrm{n}^{\circ} 1,1975-1976$, Montréal, 1976.

55. Ainsi, en 1946, au moment de la constitution du Conseil supérieur du Bien-être des familles, il donne une conférence sur l'aspect social des problèmes familiaux et dirige la discussion qui mène à la fondation de cet organisme voué à la "défense et (à) la protection sociale de la famille ": Richard Thivierge, "Un Conseil supérieur du Bienêtre des familles ", La Famille, 1946, t. X, n 6, p. 216.

56. Robert Rumilly, Maurice Duplessis et ..., t. 1, p. 668 et 669.

57. Richard Thivierge, "L'assistance à l'enfant sans soutien du diocèse des Trois-Rivières, La Famille, mai 1941, t. IV, nº 9, p. 264-269; Plante, "Une réussite aux TroisRivières... ", p. 314-317.

58. Loi relative aux écoles de protection de la jeunesse, S.Q. 1950, c. 11 ; Loi modifiant la Loi des écoles de protection de la jeunesse, S.Q. 1950-51, c. 56 ; Loi de la protection de la jeunesse, S.Q. 1959-60, c. 42.

59. Voir à ce sujet : Renée joyal et Mario Provost, "La Loi sur la protection de la jeunesse de 1977. Une maturation laborieuse, un texte porteur", (1993) 34, Les Cahiers de droit, p. 635-677. 